\title{
Multimode excitation-induced phase shifts in intrinsic Fabry-Perot interferometric fiber sensor spectra
}

\author{
Cheng $\mathrm{Ma}^{*}$ and Anbo Wang \\ Center for Photonics Technology, Bradley Department of Electrical and Computer Engineering, \\ Virginia Polytechnic Institute and State University, Blacksburg, Virginia 24061-0111, USA \\ ${ }^{*}$ Corresponding author: cma1@vt.edu
}

Received 30 June 2010; accepted 22 July 2010; posted 26 July 2010 (Doc. ID 130943); published 31 August 2010

\begin{abstract}
We report the modal analysis of optical fiber single-mode-multimode-single-mode intrinsic Fabry-Perot interferometer sensors. The multimode nature of the Fabry-Perot cavity gives rise to an additional phase term in the spectrogram due to intermodal dispersion-induced wavefront distortion, which could significantly affect the cavity length demodulation accuracy. By using an exact model to analyze the modal behavior, this phase term is explained by employing a rotating vector approach. Comparison of the theoretical analysis with experimental results is presented. (C) 2010 Optical Society of America

OCIS codes: $\quad 060.2310,060.2370,120.2230,120.3180,030.4070$.
\end{abstract}

\section{Introduction}

Fiber-optic Fabry-Perot (FP) interferometric sensors have been extensively studied in the past two decades for measurement of a variety of parameters, such as temperature [1], strain $[2, \underline{3}]$, pressure $[4,5]$, and acoustic waves [6,7]. Generally, fiber FP sensors can be divided into extrinsic and intrinsic types. In an extrinsic structure, the light from the lead-in fiber exits the fiber to propagate through a small air gap to a reflector and then reenters the fiber. The reflections from the fiber end-air interface and the reflector along with the air gap form the FP cavity. The structure can be designed to relate the gap length to various measurands for sensing.

In contrast with the extrinsic structure, the reflections in an intrinsic FP sensor are generated inside the fiber. These reflectors can be Bragg gratings [으, 9 ], reflective films [10], and air bubbles [11]. Lately, another method was proposed for the fabrication of intrinsic Fabry-Perot interferometer (IFPI) sensors [12]. In this method, the partial reflectors are produced by fusion splicing the single-mode fiber (SMF) to multimode fiber (MMF) with a well-con-

0003-6935/10/254836-10\$15.00/0

(C) 2010 Optical Society of America trolled splicing condition. The relatively large refractive index difference between the cores of the SMF and the MMF can generate quality reflections. At the same time, the light in the MMF is still guided so the insertion loss of the sensor can be controlled at a low level. This structure offers the advantages of high operating temperature and low insertion loss at the same time, which allows multiplexing a number of such sensor elements in one fiber for measurement at high temperatures. White-light interferometry has proved to be perhaps the most practical way for the demodulation of fiber F-P sensors. Typical signal demodulation for low-finesse cavities aims at finding the optical path difference (OPD), defined as OPD $=2 n L$, where $n$ is the refractive index and $L$ is the physical length of the cavity according to the sinusoidal spectral information given by [13-15]

$$
I \propto \cos (k \mathrm{OPD}+\pi+\varphi) .
$$

In Eq. (1), the DC component has already been removed. The sinusoidal nature of the spectrum comes from the interference generated by the two cavity mirrors. $k$ is equal to $2 \pi / \lambda$, where $\lambda$ is the light wavelength in vacuum, the term $\pi$ comes from the reflection from an optically denser medium, and the term $\varphi$ 
is an additional phase term, which has been investigated thoroughly in the case of SMF extrinsic FabryPerot interferometers (EFPIs) and MMF EFPIs by Han et al. $[16,17]$. This additional phase term is explained as a result of wavefront distortion of the light during propagation in free space. The demodulation of the sensor's OPD can be performed either by the peak-tracking method [13] or the phase linear regression method [15]. The OPD demodulation accuracy can be greatly enhanced by measuring the additional phase term $\varphi$ in advance (the so-called OPD demodulation with known phase), which requires the phase term to be a constant for any given FP cavity [15]. In reality, the phase term is dependent on the cavity length in SMF EFPI [16] and is further dependent on the mode-field distribution (MFD) in the lead-in MMF for MMF EFPI [17], which means one needs to be very cautious before assuming the phase term to be constant during OPD measurement. By taking into account the overall effect exerted by the multiple transmission modes in the cavity, we analyzed the interference spectrogram of the single mode-multimode-single-mode (SMS) IFPI sensor and concluded that a nonconstant phase term also existed. Similar to the EFPI case, the phase term was generated by the field wavefront distortion; however, for EFPIs, the distortion comes from free-space propagation, while for IFPIs it is a side effect of intermodal dispersion.

\section{Modal Analysis of the SMS IFPI}

\section{A. Exact Field Expression}

A typical SMS IFPI sensor is fabricated by fusion splicing a lead-in SMF to a section of MMF; the MMF is cleaved to a desired length (cavity length $L$ ) and spliced using the same splicing condition to a leadout SMF. The interfaces at the two splicing points generate weak light reflections due to the difference in the core refractive indices of the SMF and MMF. Figure 1 shows the geometry of the SMS IFPI sensor.

Instead of applying Eq. (1) to obtain the sensor spectrum, a more accurate model employs analysis of modal coupling and propagation in the multimode waveguide [18-21]. Light in the SMF is carried by the $\mathrm{LP}_{01}$ mode of the SMF with normalized mode profile $\Phi_{0}$. At the interface $R_{1}$, the mode energy is split into several parts carried separately by $\mathrm{LP}_{0 k}$ modes with normalized mode profile $\Phi_{k}$ in the MMF (here we made the assumption that the MMF is a weakly guided, step-index fiber for simplicity of analysis). Modes in the MMF with azimuthal number other

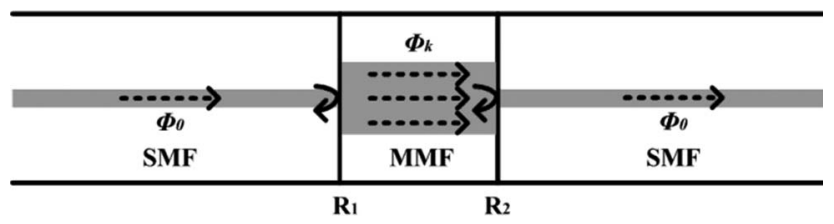

Fig. 1. Schematic of an SMS-IFPI sensor. than zero will not be exited due to mode orthogonality. For each mode, the coupling coefficient is defined as $\eta_{0 \rightarrow k}=\iint_{R_{1}} \phi_{0} \phi_{k}^{*} \mathrm{~d} x \mathrm{~d} y$. Being reflected by $R_{2}$ and propagating to $R_{1}$, the $k$ th mode profile can be expressed as $r \eta_{0 \rightarrow k} \phi_{k} \exp \left[j\left(\frac{2 \pi}{\lambda} \mathrm{OPD}_{k}+\pi\right)\right]$, where $\mathrm{OPD}_{k}=2 n_{k} L$ and $n_{k}$ is the effective index of the $k$ th mode. At interface $R_{1}$, all these modes will be recoupled into the fundamental $\mathrm{LP}_{01}$ mode of the SMF with coupling coefficient $\eta_{k \rightarrow 0}=\iint_{R_{1}} \phi_{k} \phi_{0}^{*} \mathrm{~d} x \mathrm{~d} y=$ $\eta_{0 \rightarrow k}^{*}$. As a result, the contribution to the total mode profile by each mode can be described as $r\left|\eta_{0 \rightarrow k}\right|^{2} \phi_{0} \exp \left[j\left(\frac{2 \pi}{\lambda} \mathrm{OPD}_{k}+\pi\right)\right]$, and the total field profile at $R_{1}$ can be expressed as

$$
\phi_{s}=r \phi_{0}\left(1+\sum_{k=1}^{N} \eta_{k}^{2} \exp \left[j\left(\frac{2 \pi}{\lambda} \mathrm{OPD}_{k}+\pi\right)\right]\right)
$$

where $\eta_{k}=\left|\eta_{0 \rightarrow k}\right|$ is a real number and $N$ modes are assumed to be excited in the MMF. Based on the expression for the total field above, we can find the reflection intensity from the sensor as

$$
I=\iint_{R_{1}} \phi_{s} \phi_{s}^{*} \mathrm{~d} x \mathrm{~d} y=r^{2}|1+\Sigma|^{2},
$$

where $\Sigma=\sum_{k=1}^{N} \eta_{k}^{2} \exp \left[j\left(\frac{2 \pi}{\lambda} \mathrm{OPD}_{k}+\pi\right)\right]$ is an effective vector to be discussed in more detail in Subsection 2. C. In addition, we have already applied the normalization condition $\iint_{R_{1}} \phi_{0} \phi_{0}^{*} \mathrm{~d} x \mathrm{~d} y=1$. Note that, if only one mode is excited in the MMF, Eq. (3) will be automatically reduced to Eq. (1) after canceling the DC component.

\section{B. Two-Mode Excitation}

The expression for the total field intensity deduced from the last section [Eq. (3)] is comprehensive but relatively complicated. Here we consider a much simpler case in which only two modes are excited in the MMF with similar effective refractive indices; this analysis can provide us with an illustrative understanding of how intermodal dispersion affects the sensor spectrum.

Assuming that the effective indices of the two modes are $n_{1}$ and $n_{2}$, respectively, and, accordingly, the OPD experienced by the two modes are $\mathrm{OPD}_{1}=$ $2 n_{1} L$ and $\mathrm{OPD}_{2}=2 n_{2} L$, we define $\Delta \mathrm{OPD}=\mathrm{OPD}_{1}-$ $\mathrm{OPD}_{2}$ such that $\mathrm{OPD}_{2}$ can be expressed by $\mathrm{OPD}_{2}=$ $\mathrm{OPD}_{1}-\Delta \mathrm{OPD}$.

The total reflected spectrum can be expressed as

$$
\begin{aligned}
I= & 1+\eta_{1}^{4}+\eta_{2}^{4}+2 \eta_{1}^{2} \cos \left(\mathrm{OPD}_{1} \bullet k_{0}\right) \\
& +2 \eta_{2}^{2} \cos \left(\left(\mathrm{OPD}_{1}-\Delta \mathrm{OPD}\right) \bullet k_{0}\right) \\
& +2 \eta_{1}^{2} \eta_{2}^{2} \cos \left(\Delta \mathrm{OPD} \bullet k_{0}\right) .
\end{aligned}
$$

In Eq. (4), the fourth and fifth terms on the righthand side describe the interference of the modes with 
themselves, and the last term accounts for the interference between the two modes. After some mathematical manipulations, Eq. (4) can be rewritten as

$I=r^{2}\left\{1+\Gamma+2 \sqrt{\Gamma} \sin \left[\mathrm{OPD}_{1} k_{0}+\varphi\left(\Delta \mathrm{OPD}, k_{0}\right)+\pi\right]\right\}$,

where
$\Sigma$ is defined as $\Sigma=\sum_{k=1}^{N} \eta_{k}^{2} \exp \left[j\left(\frac{2 \pi}{\lambda} \mathrm{OPD}_{k}+\pi\right)\right]$. The received power can be expressed as

$$
\begin{aligned}
I & \propto|1+\Sigma|^{2}=1+|\Sigma|^{2}+2|\Sigma| \cos (\psi), \quad \text { where } \psi \\
& =\angle \Sigma .
\end{aligned}
$$

Equation (6) leads to the conclusion that the total phase in the interference spectrogram can be directly

$$
\begin{aligned}
\Gamma=\eta_{1}^{4}+\eta_{2}^{4}+2 \eta_{1}^{2} \eta_{2}^{2} \cos \left(\Delta \mathrm{OPD} \bullet k_{0}\right) \\
\varphi\left(\Delta \mathrm{OPD}, k_{0}\right)=\left\{\begin{array}{c}
\arctan \left(\frac{\eta_{1}^{2}+\eta_{2}^{2} \cos \left(\Delta \mathrm{OPD} \bullet k_{0}\right)}{\eta_{2}^{2} \sin \left(\Delta \mathrm{OPD} \bullet k_{0}\right)}\right), \sin \left(\Delta \mathrm{OPD} \bullet k_{0}\right) \geq 0 \\
\pi-\arctan \left(\frac{\eta_{1}^{2}+\eta_{2}^{2} \cos \left(\Delta \mathrm{OPD} \bullet k_{0}\right)}{\left|\eta_{2}^{2} \sin \left(\Delta \mathrm{OPD} \bullet k_{0}\right)\right|}\right), \sin \left(\Delta \mathrm{OPD} \bullet k_{0}\right)<0
\end{array}\right.
\end{aligned}
$$

It is not difficult to find that, at the limit $\eta_{2}^{2} \ll \eta_{1}^{2}$ (which means the second mode is excited so weakly that almost all the power is carried by the first mode), Eq. (5) reduces to Eq. (1) with the additional phase term equal to zero. As the second mode emerges, the amplitude and phase of the sinusoid will be modulated. The phase term $\varphi\left(\Delta \mathrm{OPD}, k_{0}\right)$ as a function of $\Delta \mathrm{OPD} \bullet k_{0}$ has been plotted in Fig. 2 . To plot the figure, the wavelength span was set from 1400 to $1700 \mathrm{~nm}$, the effective refractive indices of mode 1 and mode 2 were set to be 1.448 and 1.441 , respectively, the cavity length was set to $1 \mathrm{~mm}$, and four different cases with excitation ratio $\left(\eta_{1}^{2} / \eta_{2}^{2}\right)$ of $6: 4,7: 3,8: 2$, and $9: 1$ were compared.

\section{Rotating Vector Picture}

In Subsection 2.B, we only considered the excitation of two modes inside the MMF cavity. In reality, the number of modes excited can be much more, which complicates the problem. From Eq. (3), the received intensity is proportional to $|1+\Sigma|^{2}$, where the vector

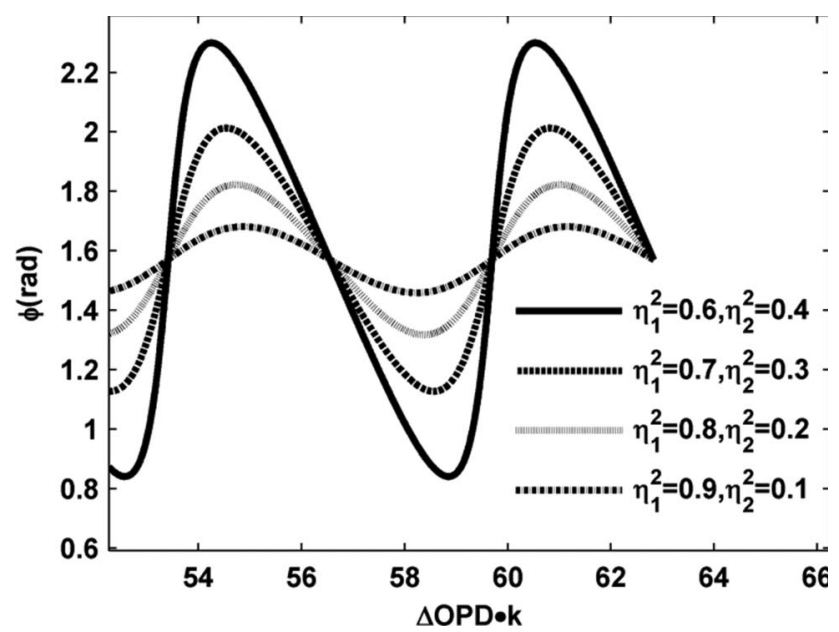

Fig. 2. Additional phase term for a two-mode cavity at different excitation ratios. obtained from the angle of the vector $\Sigma$. In the following discussion, for simplicity without losing generality, we omit the phase term $\pi$ in the expression of vector $\Sigma$ :

$$
\Sigma=\sum_{k=1}^{N} \eta_{k}^{2} \exp \left[j\left(\frac{2 \pi}{\lambda} \mathrm{OPD}_{k}\right)\right]
$$

where $\mathrm{OPD}_{k}=2 n_{k} L$ and $n_{k}$ is the effective refractive index of the $k$ th mode. It is straightforward to find out that $\Sigma$ is the sum of $N$ vectors representing $N$ orthogonal modes (we define the mode vector as $\left.v_{k}=\eta_{k}^{2} \exp \left[j\left(\frac{2 \pi}{\lambda} \mathrm{OPD}_{k}\right)\right]\right)$; the modulus of each vector is the square of the magnitude of the mode coupling coefficient between the given mode and the $\mathrm{LP}_{01}$ mode of the SMF; the phase of each vector is simply the product of the vacuum wavenumber and the effective OPD of that mode. Figure 3 illustrates how the total field vector is related to each individual mode vector. In total, five modes were assumed to be excited.

In the interference spectrogram, as the wavenumber $k$ increases, all the $N$ individual mode vectors $\left(v_{k}\right)$ will rotate at different angular speed $\mathrm{OPD}_{k}=2 n_{k} L$. The mode vector of the fundamental $\mathrm{LP}_{01}$ mode rotates at the fastest speed (corresponding to the largest effective refractive index), while the mode vector of $\mathrm{LP}_{0 N}$ mode rotates the slowest. The differences among rotational speeds of all the mode vectors

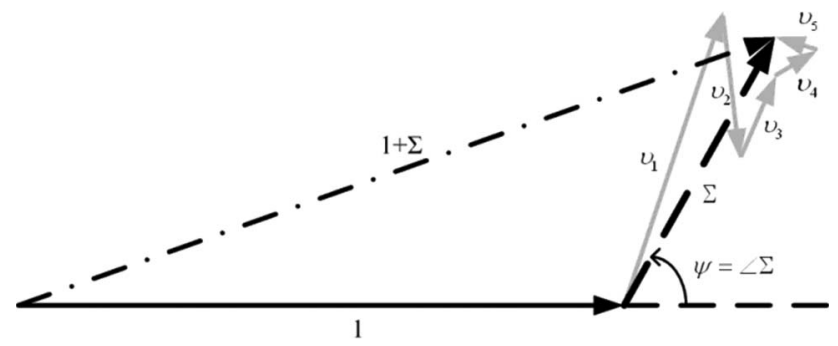

Fig. 3. Relationship between the total field vector and the five individual mode vectors. 
are very small because modal refractive indices have very similar values. Although the individual vectors rotate at similar speeds, their pointing directions are almost arbitrary because the wavenumber $k$ and the cavity length $L$ are both very large numbers. The effective vector $\Sigma$ rotates at a speed that depends simultaneously on the rotational speed of each vector and their relative orientations. If most of the mode vectors align at similar directions, the rotating speed of the effective vector can be slower than any of the individual vectors; there are also occasions where its rotational speed is faster than any of the individual vectors (see Fig. 4 for an example).

Figure 4 plots the mode vectors of all seven excited modes in a step-index MMF with $n_{\text {core }}=1.448$, $n_{\text {clad }}=1.434$, and $r_{\text {core }}=25 \mu \mathrm{m}$, excited by SMF with $n_{\text {core }}=1.445, n_{\text {clad }}=1.440$, and $r_{\text {core }}=4.2 \mu \mathrm{m}$. In the simulation, the cavity length is $2 \mathrm{~mm}$, and the wavelength is set to be $\lambda=1550 \mathrm{~nm}$. Increasing wavenumber $k$ results in rotation of mode vectors, while the effective vector rotates correspondingly. In total, nine groups of vectors are plotted with equal angular spacing, such that the fundamental mode vector $\left(v_{1}\right)$ of the ninth group coincides with that of the first group, which means by increasing $k, v_{1}$ rotates exactly $2 \pi$. Since the fundamental mode travels the fastest, all the higher-order modes rotate at slower rates; none of the higher-order modes rotates as far as $2 \pi$. However, the effective vector rotates faster than the fundamental mode vector, as shown in Fig. 4. The end of the cascaded gray arrows (which represents the effective vector after $v_{1}$ rotates by $2 \pi$ ) falls ahead of the end of the cascaded dark arrows (which represents the effective vector before rotation), indicating a fas-

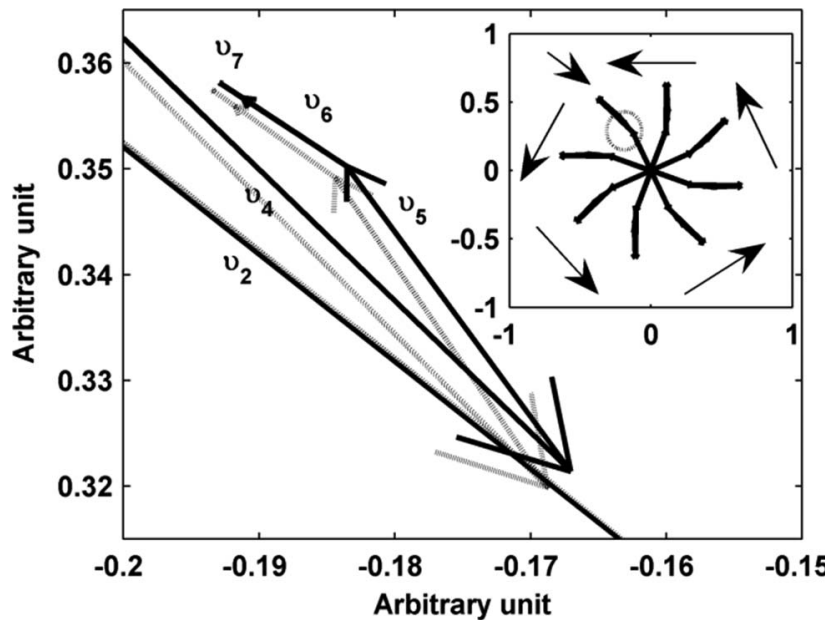

Fig. 4. Zoom-in view of the rotating vectors: Nine groups of vectors are plotted with equal angular spacing such that $v_{1}$ rotates exactly $2 \pi$. Dark lines, first group of vectors (beginning position); gray lines, last group of vectors (end position: $v_{1}$ coincides with $v_{1}$ in the first group; $v_{2}$ is a little behind $v_{2}$ in the first group; the effective vector $\Sigma$ is ahead of $\Sigma$ in the first group). Inset: global view of the vector rotation. Upper-left arrow, position of the first and last groups of vectors; other arrows illustrate the rotational direction of the vectors. ter rotational speed of the effective vector than the fundamental mode vector $v_{1}$.

As $k$ increases, all the vectors begin to rotate; while each individual mode vector rotates at constant speed, the effective vector does not, even though the speed variation is not significant if $k$ scans in a narrow range (within the spectral range of the interference spectrogram). Taking the rotational speed of $v_{1}$ as a reference, the relative speed for any other vector can be obtained by subtracting the speed of $v_{1}$ from its own speed. At any given wavenumber $k$, this relative speed is proportional to the phase difference between that vector and the $\mathrm{LP}_{01}$ vector. For the cavity we examined to plot Fig. 4, when the wavelength is scanned from 1520 to $1570 \mathrm{~nm}$, the relative phase shift (taking $\mathrm{LP}_{01}$ vector as the reference) of the following vectors are plotted in Fig. 5: $\mathrm{LP}_{02}$ (at a lower rotating speed), the effective vector $\Sigma$ (at a faster, varying speed), and the linear fitting of the rotating speed of $\Sigma$ (represents a virtual mode vector that best approximates the rotating speed of the effective vector). The angular rotating speed of the virtual vector is actually the first-step-estimated $\mathrm{OPD}^{(1)}$ of the cavity, as is discussed in Section $\underline{3}$.

\section{Results and Discussion}

In an actual sensor application, a change in the measurand, such as strain or temperature, varies the OPD of the SMS-IFPI sensor; by detecting with high accuracy the OPD change, the change in the measurand can be decoded correspondingly. For example, strain changes the OPD of the cavity as

$$
\frac{\Delta \mathrm{OPD}}{\mathrm{OPD}}=\left(1-p_{e}\right) \varepsilon
$$

where $p_{e}$ is the effective strain-optic constant and $\varepsilon$ is the applied strain [22]. Temperature modifies both the dimension and the refractive indices of the cavity according to

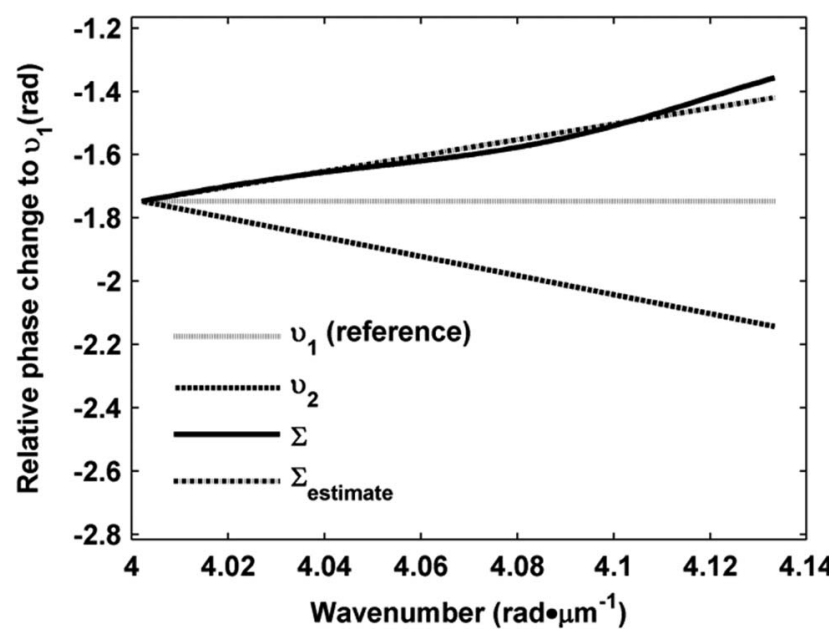

Fig. 5. Relative phase shift to $\mathrm{LP}_{01}$ mode as $k$ increases. Plotted are phase shifts of $\mathrm{LP}_{02}$ mode, the effective vector $\Sigma$, and a virtual mode with $n_{\text {eff }}=n_{\text {estimate }}$. 


$$
\frac{\Delta \mathrm{OPD}}{\mathrm{OPD}}=\left(\alpha_{T}+\sigma_{T}\right) \Delta T,
$$

where $\alpha_{T}$ is the coefficient of thermal expansion (CTE) of silica, and $\sigma_{T}$ is the thermal-optic coefficient of the fiber core [22]. Hence, the key of measurement accuracy and resolution relies on the OPD decoding quality.

Most of the OPD decoding algorithms assume the sensor spectrogram to be purely sinusoidal $[14,15]$. According to Eq. (1), the total phase in the interference spectrum is

$$
\varphi_{\text {tot }}=k \bullet \mathrm{OPD}+\varphi_{0},
$$

where $\varphi_{0}$ is the additional phase term introduced in section one (the phase term $\pi$ is omitted). Equation (10) indicates that the OPD of the cavity equals the angular rotational speed of the total phase $\varphi_{\text {tot }}$ with $k$. The wavenumber $k_{m}$ corresponding to the peak locations of the interference pattern should satisfy the phase relationship: $k_{m} \bullet \mathrm{OPD}+\varphi_{0}=2 \pi m$, where $m$ is the fringe order of the corresponding peak; let us denote the smallest fringe order on the spectrogram to be $m_{0}$; we have $k_{m} \bullet \mathrm{OPD}+$ $\varphi_{0}=2 \pi\left(m_{0}+N\right)$, where $N=0,1,2, \ldots$ The above relationship can be rewritten as

$$
\begin{aligned}
k_{m} \bullet \mathrm{OPD}+\varphi_{0}^{\prime} & =2 \pi N, \quad \varphi_{0}^{\prime}=\varphi_{0}-2 \pi m_{0}, \\
N & =0,1,2, \ldots
\end{aligned}
$$

By linearly fitting $k_{m}$ with $N$, one can find the firststep estimation of the optical path difference $\mathrm{OPD}^{(1)}$. Multiple measurements can be performed to accurately find the phase term $\varphi_{0}^{\prime}$ in advance so that, for any measurement that follows, we can predict the location of the $m$ th fringe peak by

$$
k_{m}^{(1)} \bullet \mathrm{OPD}^{(1)}+\varphi_{0}^{\prime}=2 \pi N .
$$

The OPD estimation error ( $\delta \mathrm{OPD})$ is related to a phase shift $\delta \varphi$ by

$$
\delta \mathrm{OPD}=\frac{\delta \varphi}{2 \pi} \lambda_{m}
$$

By setting $\lambda_{m}=1550 \mathrm{~nm}$, one can calculate that a phase shift of $\pm \pi$ can be induced by an OPD estimation error of $\pm 775 \mathrm{~nm}$, which is very large. According to computer simulation results, at high signal-tonoise ratio $(\mathrm{SNR})>30 \mathrm{~dB}$, the first-step OPD estimation errors fall within $\pm 200 \mathrm{~nm}$, which correspond to a phase shift of $\pm 0.26 \pi$. If we predict the peak locations by inserting the first-step estimation result $\mathrm{OPD}^{(1)}$ into Eq. (12), the phase difference between the real spectrum and predicted spectrum will have a phase difference within the range $[-0.26 \pi,+0.26 \pi]$. Fig. 6 illustrates this situation.

As shown in Fig. 6, the predicted peaks appear at the vicinity of the real peaks within the phase range

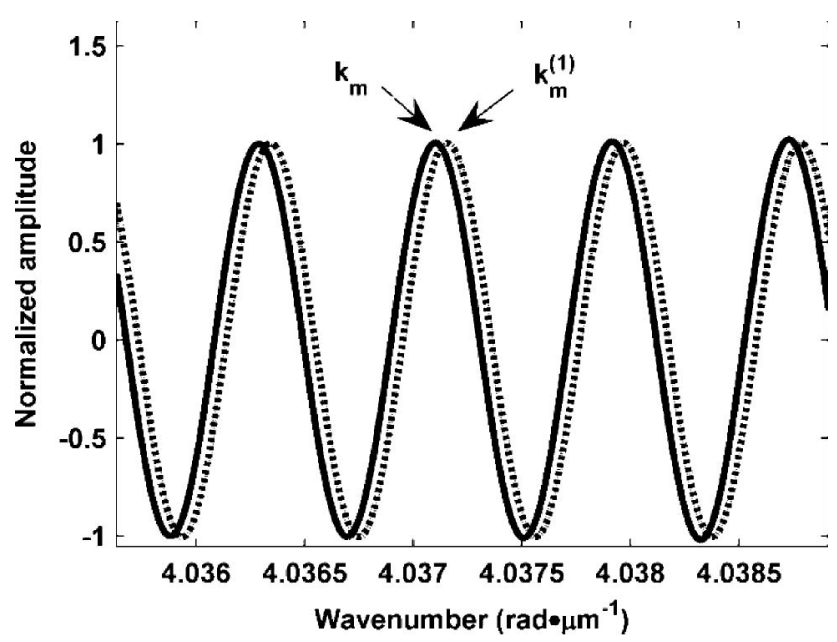

Fig. 6. Spectral phase shift induced by OPD estimation error.

$[-0.26 \pi,+0.26 \pi]$. Mathematically, this can be written as

$$
k_{m}^{(1)} \bullet \mathrm{OPD}^{(1)}=k_{m} \bullet \mathrm{OPD} .
$$

We can obtain a better estimation of the value for OPD by

$$
\mathrm{OPD}^{(2)}=\frac{k_{m}^{(1)}}{k_{m}} \bullet \mathrm{OPD}^{(1)} .
$$

This second-step estimation of the OPD has much better accuracy, according to computer simulation. The OPD estimation error is within $\pm 1 \mathrm{~nm}$.

The OPD demodulation algorithm described above assumes that the additional phase term in Eq. (10) is a constant. However, as mentioned in Section 1, this term is not a constant and can vary with the OPD, which adds to the complexity of the signal decoding. We use Eq. (3) to calculate the interference spectrogram for our computer simulation. To calculate the refractive indices of different modes in the SMF and MMF with tolerable computational complexity, we assumed that the fibers to satisfy the weakly guiding condition and the orthogonal modes supported by the fibers are linearly polarized modes (LP modes) [18]. The fiber characteristic equation used for calculation of the effective refractive index $n_{\text {eff }}$ is given by [18]

$$
\frac{J_{l}(u)}{u J_{l-1}(u)}+\frac{K_{l}(w)}{w K_{l-1}(w)}=0,
$$

where $J_{l}(x)$ is the Bessel function of the first kind, and $K_{l}(x)$ is the modified Bessel function of the second kind, $l$ corresponds to the azimuthal number of the mode (in our case $l=0$; see discussion in Section 2), $u=(2 \pi a / \lambda)\left(n_{1}^{2}-n_{\text {eff }}^{2}\right)^{1 / 2}, w=(2 \pi a / \lambda) \times$ $\left(n_{\text {eff }}^{2}-n_{2}^{2}\right)^{1 / 2}$, in which $a$ is the radius of the fiber core, and $n_{1}$ and $n_{2}$ are the refractive indices of the fiber core and cladding, respectively.

To calculate the mode coupling coefficient $\eta_{k}=\left|\iint_{R_{1}} \phi_{0} \phi_{k}^{*} \mathrm{~d} x \mathrm{~d} y\right|$, in which $\phi_{0}$ is the mode profile 
for the fundamental mode in the SMF and $\phi_{k}$ is the $k$ th mode profile in the MMF, we applied the mode profile formula [18]:

$$
\phi_{k}=\left\{\begin{array}{c}
A J_{0}\left(u_{k} r / a\right) / J_{0}\left(u_{k}\right), r<a \\
A K_{0}\left(w_{k} r / a\right) / K_{0}\left(w_{k}\right), r>a
\end{array},\right.
$$

where $k$ denotes the $k$ th root of Eq. (16) and the value of $A$ can be obtained by the normalization condition: $\left|\iint_{R_{1}} \phi_{k} \phi_{k}^{*} \mathrm{~d} x \mathrm{~d} y\right|=1$.

Another approximation used to simplify the calculation is to consider only intermodal dispersion in the MMF; the intramodal dispersion (which accounts for the fact that $n_{\text {eff }}$ of each mode also depends on wavenumber $k$ ) is ignored. This is a reasonable assumption because in real applications one obtains the spectral information only in a narrow spectral range (for example, in our simulations and experiments, from 1520 to $1570 \mathrm{~nm}$ ) in which $n_{\text {eff }}$ can be regarded as a constant.

Equation (8) will be applied for simulation of quantities that induce only fiber dimensional change while setting $p_{e}$ to a constant, and Eq. (9) will be applied for simulation of the temperature effect when dimensional and refractive indices changes coexist. The fiber parameters used for all the simulations are the following: step-index MMF with $n_{\text {core }}=$ $1.448, n_{\text {clad }}=1.434$, and $r_{\text {core }}=25 \mu \mathrm{m}$, excited by step-index SMF with $n_{\text {core }}=1.445, n_{\text {clad }}=1.440$, and $r_{\text {core }}=4.2 \mu \mathrm{m}$.

\section{A. Physical Meaning of the OPD-Dependant Phase Term}

By using Eq. (11) to estimate the OPD or, equivalently, the angular speed of the total phase with $k$, one assumes that the rotational speed (OPD) is a constant such that least-squares (LS) linear fitting gives the best estimation. As concluded in Section 2, the measurable (obtainable) rotational speed is actually represented by the rotation of the effective vector $\Sigma$. From the discussion in Section 2, the rotational speed of this vector depends on the alignments of all the mode vectors $v_{k}$. At a constant OPD, all the mode vectors $\left(v_{k}\right)$ rotate at different but constant speeds, resulting in evolution of the relative alignments of the mode vectors, and the rotational speed of vector $\Sigma$ will undergo a change as $k$ increases. This means the rotation of the effective vector has acceleration. As shown clearly in Fig. 5, the phase change is not linearly related to $k$. If we carry out LS linear fitting applying Eq. (11), instead of getting fitting errors evenly distributed around zero (which corresponds to a constant rotation speed), the fitting error shows a dispersive pattern. This is illustrated in Fig. 7.

Because the rotational speed of the effective vector is affected strongly by the relative alignments of all the mode vectors, the best one can do is to look at the spectrum and estimate its speed by linear fitting. As a result, the first-step estimation $\mathrm{OPD}^{(1)}$ can be misleading: the cavity can appear to be longer or shorter than it really is if the observation is made simply

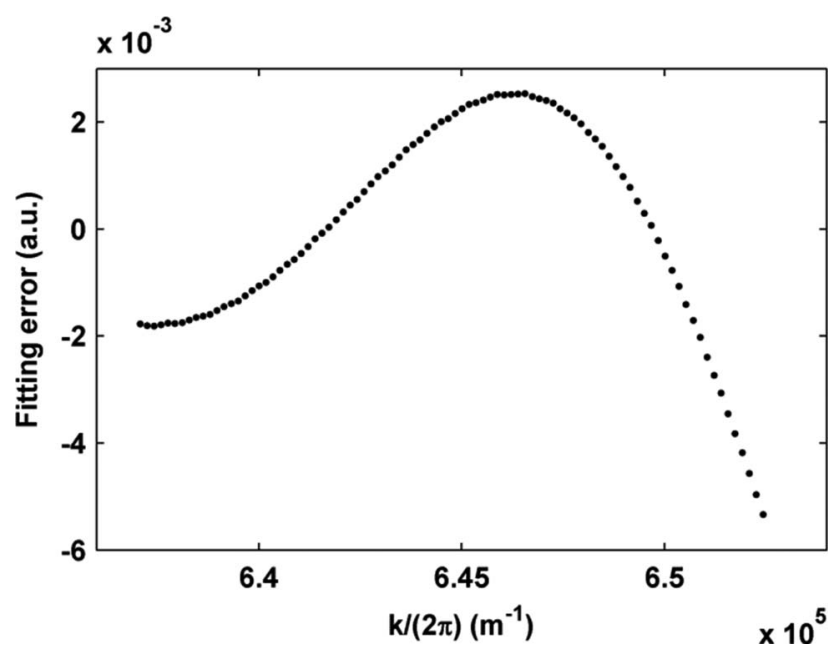

Fig. 7. Simulated linear fitting error as a function of wavenumber.

based on looking at the interference spectrogram generated by the cavity. Consequently, if Eq. (12) is used to predict the peak positions, large phase error can occur; as the OPD changes, the relative alignments of the mode vectors also change, resulting in varying "estimation quality" of the OPD, and the additional phase term will vary accordingly.

OPD change solely due to fiber dimensional change was studied by computer simulation. The cavity length was changed from 2000 to $2010 \mu \mathrm{m}$. At a given cavity length, the total phase difference between the real spectrum and predicted spectrum based on $\mathrm{OPD}^{(1)}$ was calculated; for the cavity length range studied, this phase difference was plotted as a function of the cavity length. During the simulation, no noise was added to the signal to remove phase ambiguity generated by external noise. As shown in Fig. $\underline{8}$, a nearly $-\pi$ phase change occurred during this process.

Assume that, during the above-simulated experiment, the exact value of the cavity length is known in advance. Based on the OPD estimation result, we

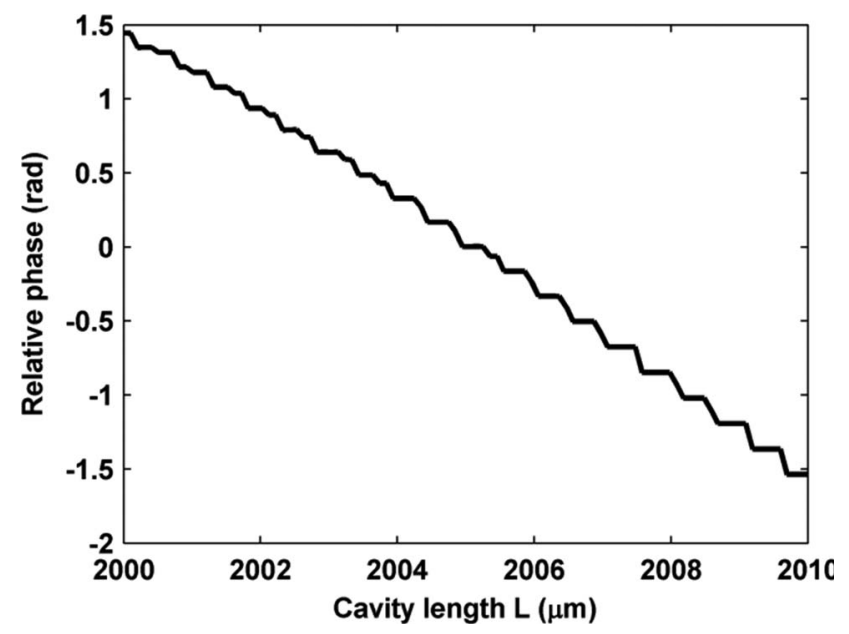

Fig. 8. Computer-simulated relative phase change as cavity length increases. 
are able to calculate the effective index of the cavity by $n_{\text {eff }}=\mathrm{OPD}^{(1)} /(2 L)$, where $\mathrm{OPD}^{(1)}$ is the first-step OPD estimation, and $L$ is the physical cavity length. In principle, the value of $n_{\text {eff }}$ should not vary during the simulation process because all the refractive indices were set to constants; however, as discussed previously, the information obtained by looking at the spectrum can be misleading because the estimation of $n_{\text {eff }}$ indeed varied a lot. In Fig. 9, the estimated $n_{\text {eff }}$ was plotted as the cavity length increases from 500 to $2500 \mu \mathrm{m}$, while all the refractive indices of the fibers were kept constant. As shown in the figure, the variation in the measured effective refractive index is significant in this process.

Based on Eq. (6), the total phase can be expressed as

$$
\varphi=\angle \Sigma=\angle\left\{\sum_{k=1}^{N} \eta_{k}^{2} \exp \left[j\left(2 k L n_{k}\right)\right]\right\} .
$$

We can approximate the rotational speed of the total phase with respect to $k$ by

$$
\frac{d \varphi}{d k}=\frac{d \varphi}{d(k L)} \frac{d(k L)}{d k} \approx 2 \bar{n}_{\mathrm{est}} L=\mathrm{OPD}^{(1)}
$$

where $\bar{n}_{\text {est }}$ is the estimation of the effective refractive index. From Eq. (19), we have $d \varphi / d(k L)=2 \bar{n}_{\text {est }}$. Also, similar to Eq. (19), the following equation can be obtained:

$$
\frac{d \varphi}{d L}=\frac{d \varphi}{d(k L)} \frac{d(k L)}{d L} \approx 2 \bar{n}_{\mathrm{est}} k
$$

Based on Eq. (20), we have

$$
\begin{aligned}
d \varphi \approx d\left(2 k \bar{n}_{\mathrm{est}} L\right) & =2 k\left(L d \bar{n}_{\mathrm{est}}+\bar{n}_{\mathrm{est}} d L\right) \\
& \approx 2 k \bar{n}_{\mathrm{est}} d L .
\end{aligned}
$$

The total phase term can be estimated with better accuracy to be

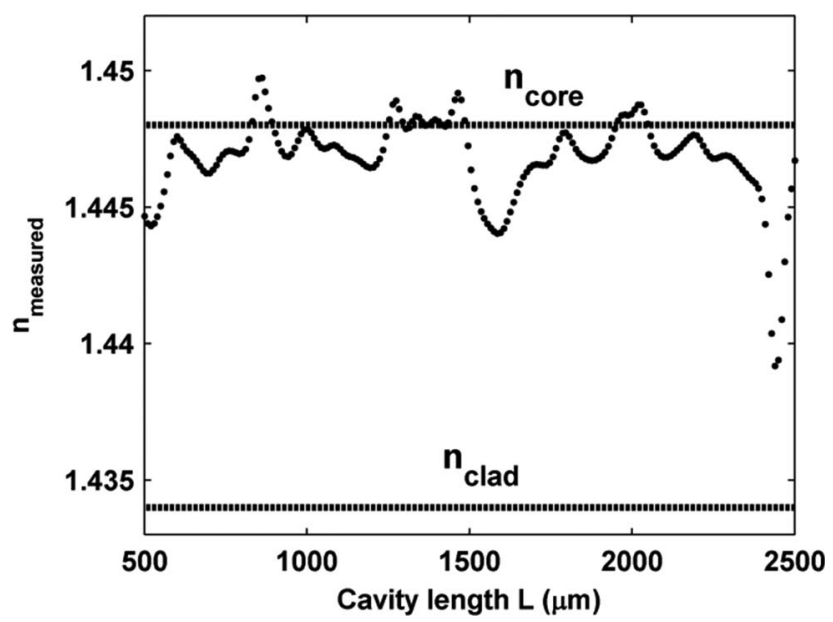

Fig. 9. Estimated $n_{\text {eff }}$ as the cavity length increases while all the refractive indices stay unchanged. Two horizontal lines mark the refractive indices of the MMF core and cladding.

$$
\varphi_{1} \approx \int_{0}^{L} 2 k \bar{n}_{\mathrm{est}} \mathrm{d} l,
$$

while the total phase is routinely predicted by

$$
\varphi_{2}=2 k \bar{n}_{\mathrm{est}} L
$$

The relationship between $\varphi_{1}$ and $\varphi_{2}$ can be graphically illustrated as shown in Fig. 10. Because the increasing rate of the total phase is not constant, using Eq. (23) to estimate the total phase will result in an additional phase term, and this error term keeps changing with increasing $L$. If the OPD change during the full measurement is small, the slope of the total phase change in that range can be regarded as constant, whereas the additional phase term will change very little accordingly; for applications where the change of OPD is large so that the slope can no longer be treated as constant, the additional phase term will change with OPD. The total phase difference is illustrated in Fig. 10 according to Eqs. (22) and (23).

From Fig. 10, the OPD-dependent phase term appears as a result of the nonconstant slope of the $\varphi-L$ curve, which is equivalent to a nonconstant $\bar{n}_{\text {est }}$. Based on Eqs. (22) and (23), the phase estimation difference as a function of cavity length $L$ is plotted in Fig. 11. To emphasize the relationship between the phase difference and the nonconstant value of the estimated $n_{\text {eff }}$, the range of $L$ is set to the same as that in Fig. 9. Shown clearly in Fig. 11 is the strong relationship between the change rate of the additional phase and the value of the estimated $n_{\text {eff. }}$. At regions where $n_{\text {eff }}$ changes rapidly, the additional phase term also changes rapidly. Also evident from Fig. 11 is the fact that there are regions where the phase changes very slowly. These regions are good for sensor application. There are also bad regions in which the phase changes rapidly; working in such regions will lead to large errors and even measurement failure.

Not only the physical dimension but also the refractive indices of the MMF will be varied during temperature measurement, which adds an additional complexity problem to the sensor signal demodulation. To have a better understanding of the

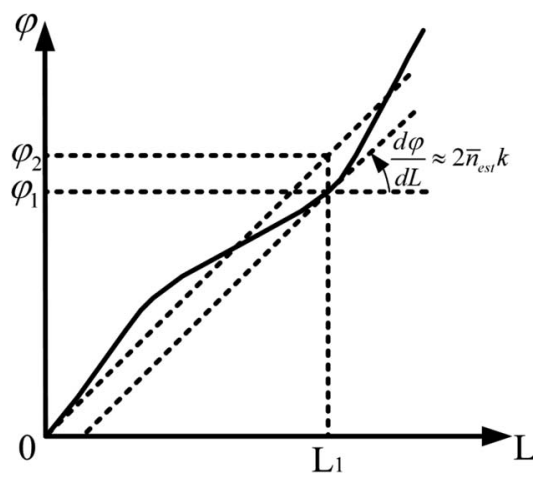

Fig. 10. Graphic explanation of the additional phase term. 


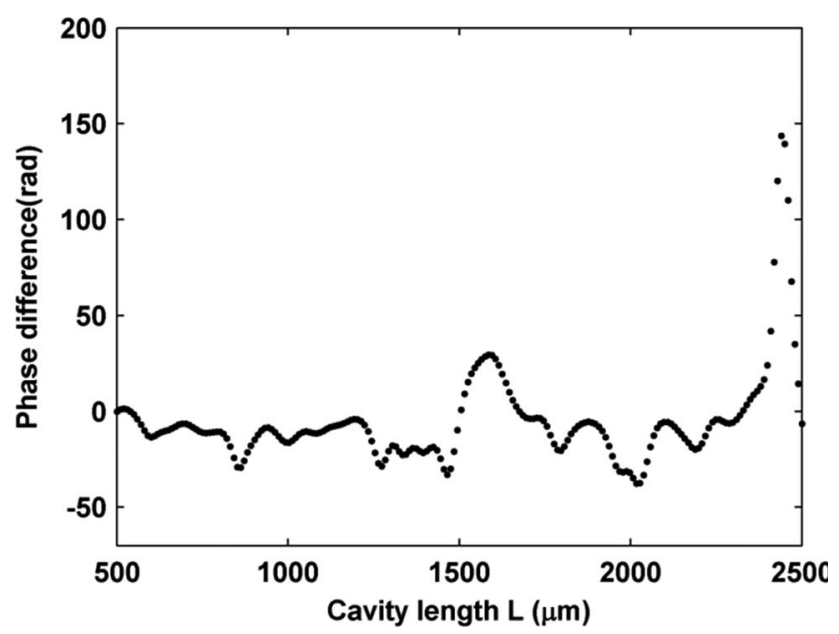

Fig. 11. Phase difference as a function of cavity length.

temperature-dependent phase term, a similar procedure can be followed as in the previous example. By employing Eqs. (9) and (19), we get

$$
\frac{d \varphi}{d T}=\frac{d \varphi}{d \mathrm{OPD}^{(1)}} \frac{d \mathrm{OPD}^{(1)}}{d T} \approx k\left(\alpha_{T}+\sigma_{T}\right) \mathrm{OPD}^{(1)} .
$$

It is straightforward to obtain a better estimation of the phase term by integrating Eq. (24):

$$
\left.\Delta \varphi_{1}\right|_{T_{1}} ^{T_{2}} \approx k\left(\alpha_{T}+\sigma_{T}\right) \int_{T_{1}}^{T_{2}} \operatorname{OPD}^{(1)}(T) \mathrm{d} T .
$$

This estimation is more accurate than the direct estimation by

$$
\left.\Delta \varphi_{2}\right|_{T_{1}} ^{T_{2}}=k\left(\mathrm{OPD}^{(1)}\left(T_{2}\right)-\mathrm{OPD}^{(1)}\left(T_{1}\right)\right) .
$$

To simulate the temperature-induced phase shift, a computer-simulated experiment was performed

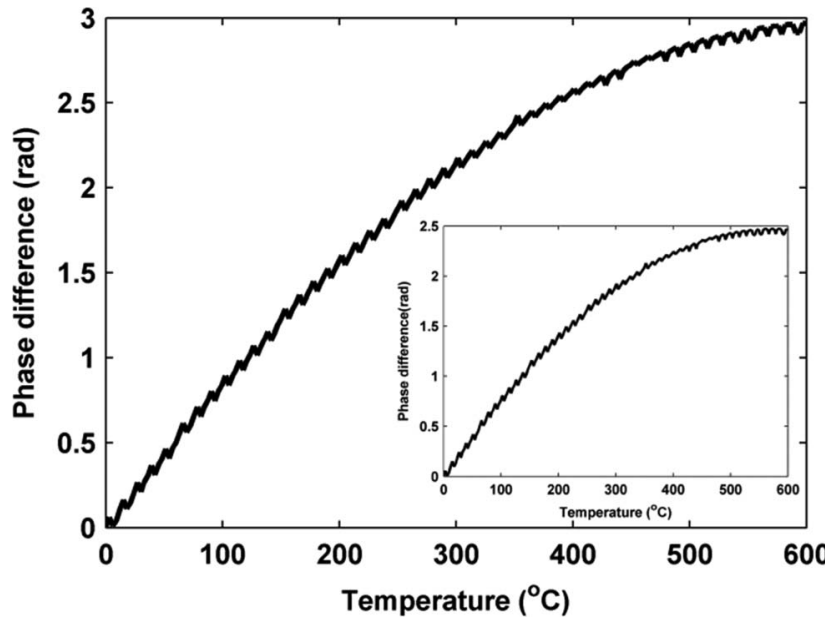

Fig. 12. Phase difference as a function of temperature, measured by direct comparison of the predicted spectrum and the real spectrum. Inset: measured by using Eqs. (25) and (26).

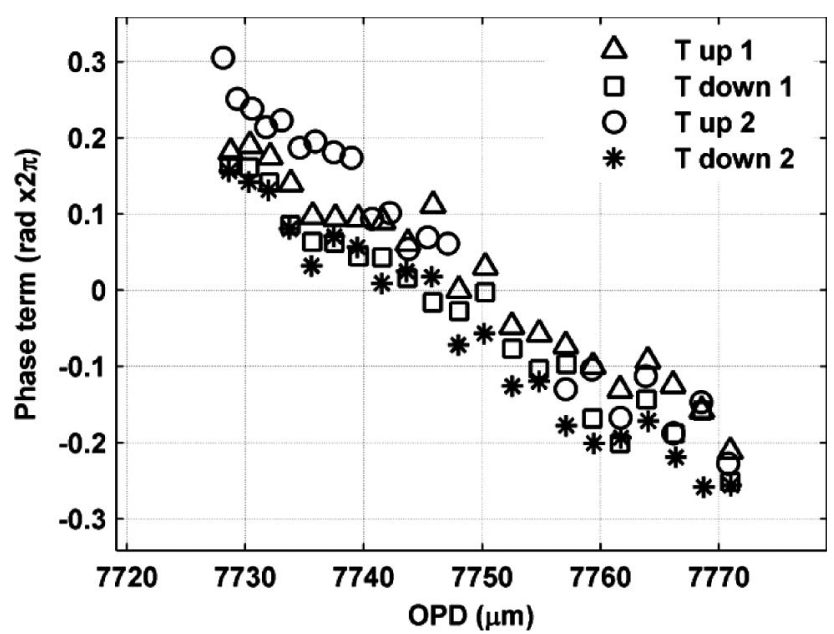

Fig. 13. Additional phase term as a function of OPD.

using a $4 \mathrm{~mm}$ cavity, the temperature was increased to $600{ }^{\circ} \mathrm{C}$ from room temperature, and two methods were applied to obtain the total phase change as a function of temperature. The first approach compared the real phase with the predicted phase [by inserting OPD ${ }^{(1)}$ into Eq. (10)], and the second approach directly compared the value of Eqs. (25) and (26). The results are provided in Fig. 12. The results obtained by these two approaches agree well except for a little discrepancy in their magnitudes.

\section{Experimental Demonstration}

An SMS-IFPI sensor was fabricated by fusion splicing a section of MMF (Thorlabs, MMF 625) sandwiched between two SMFs (Corning SMF-28). The OPD of the sensor was measured to be around $7730 \mu \mathrm{m}$. The reflection spectrum of the sensor was monitored by a spectrometer (Component Testing System, Micron Optics Inc., Si-720) from 1520 to $1570 \mathrm{~nm}$ with $2.5 \mathrm{pm}$ spectral resolution. The sensor was heated by a homemade minifurnace; the temperature of the sensor was measured by a thermocouple with $0.1^{\circ} \mathrm{C}$ resolution. The temperature was

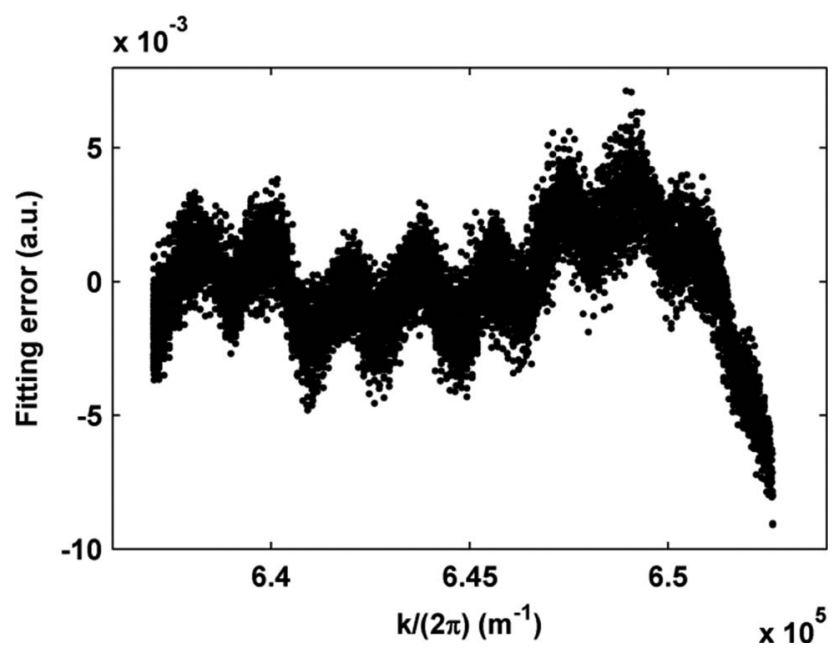

Fig. 14. Linear fitting error as a function of wavenumber (experimental). 


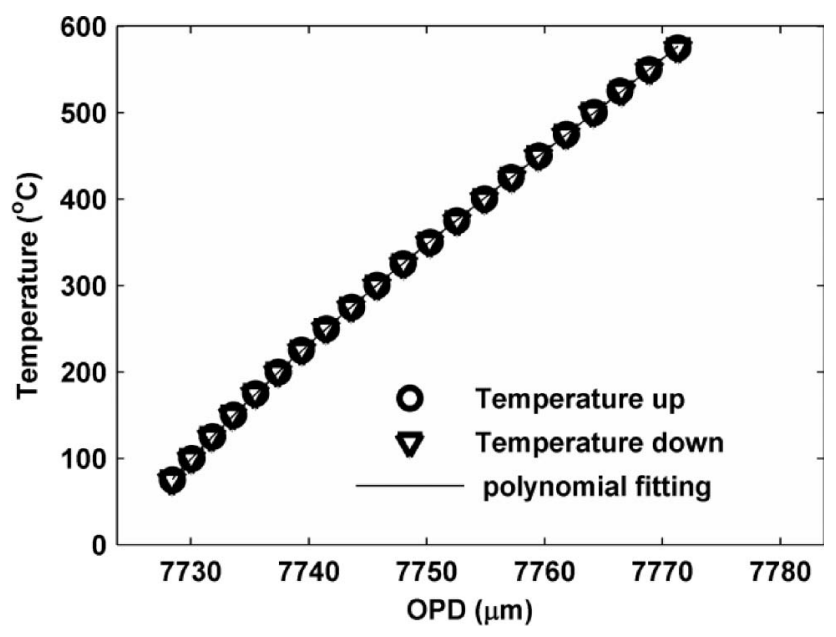

Fig. 15. Measured temperature change as a function of OPD.

controlled to scan continuously from room temperature to $600^{\circ} \mathrm{C}$ multiple times, and interference spectra were obtained at $2.5^{\circ} \mathrm{C}$ intervals. From each spectrogram recorded, the first-step OPD estimation was performed to obtain $\mathrm{OPD}^{(1)}$, and based on the first-step predicted value, the total phase was estimated and compared with the phase of the real data to calculate the additional phase term. Figure 13 plots the additional phase term as a function of OPD. A phase change up to $-\pi$ can be seen in the figure.

Figure 14 shows the fitting error as a function of wavenumber for 90 groups of experimental data. As predicted by the theory, the fitting error manifests a certain dispersive pattern. Although the pattern obtained from the real sensor shows more ripples than the simulation result (Fig. 7), the pattern itself shows agreement with the prediction of the theory. According to Fig. 13, the phase term could be linearly fitted with OPD as $\Delta \varphi(\mathrm{OPD})=\pi(153.85-$ $20013 \times$ OPD); this term can be added into Eq. (12) to precalibrate the sensor for spectral peak position calculation to obtain second-step OPD estimation. The estimated $\mathrm{OPD}^{(2)}$ as a function of temperature is plotted in Fig. 15 .

\section{Conclusion}

Wavefront distortion in the MMF section of an SMSIFPI sensor is a direct result of the multimodal nature of the cavity. Similar to EFPI fiber sensors, the wavefront distortion generates an additional phase term in the interference spectrogram of the sensor. The total phase in the spectrogram is modeled by a rotating effective vector. The rotation of the vector always happens at a nonconstant speed with wavenumber, resulting in a spectrum that seems denser (corresponding to a faster rotation speed) or sparser (slower rotation speed) than it is supposed to be by the traditional treatment of the cavity as single mode. Mathematically, the OPD demodulation process is the estimation of the rotational speed of the effective vector with respect to wavenumber by looking at the interference spectrum; however, the infor- mation obtained herein can be misleading to some extent. Hence, the error in this first-step estimation amounts to an additional phase shift in the formula we used to calculate the total phase of the spectrogram. It is predicted that in a real application, if the OPD change is small in a measurement, the change of the additional phase term will not be significant and high measurement accuracy can still be obtained; however, for applications where OPD changes are large, there exist either good or bad zones. For those good zones, the OPD-dependent additional phase term will not change much during the full measurement range so that the phase term can be precalibrated (as shown in Section 4); however, for the bad zones, the phase term will change very rapidly and accurate OPD decoding will be difficult.

This work is funded by the U.S. Department of Energy (DOE) under grant DE-NT0005591. The authors are grateful for helpful discussions with Ming Han in the Department of Electrical Engineering at the University of Nebraska-Lincoln.

\section{References}

1. C. E. Lee and H. F. Taylor, "Fiber-optic Fabry-Perot temperature sensor using a low-coherence light source," J. Lightwave Technol. 9, 129-134 (1991).

2. C. Belleville and G. Duplain, "White-light interferometric multimode fiber-optic strain sensor," Opt. Lett. 18, 78-80 (1993).

3. K. A. Murphy, M. F. Gunther, A. M. Vengsarkar, and R. O. Claus, "Quadrature phase-shifted, extrinsic Fabry-Perot optical fiber sensors," Opt. Lett. 16, 273-275 (1991).

4. A. Wang, H. Xiao, J. Wang, Z. Wang, W. Zhao, and R. G. May, "Self-calibrated interferometric-intensity-based optical fiber sensors," J. Lightwave Technol. 19, 1495-1501 (2001).

5. Y. Kim and D. P. Neikirk, "Micromachined Fabry-Perot cavity pressure transducer," IEEE Photonics Technol. Lett. 7, 1471-1473 (1995).

6. N. Furstenau, M. Schmidt, H. Horack, W. Goetze, and W. Schmidt, "Extrinsic Fabry-Perot interferometer vibration and acoustic sensor systems for airport ground traffic monitoring," IEE Proc. Optoelectron. 144, 134-144 (1997).

7. J. F. Dorighi, S. Krishnaswamy, and J. D. Achenbach, "Stabilization of an embedded fiber optic Fabry-Perot sensor for ultrasound detection," IEEE Trans. Ultrason. Ferroelectr. Freq. Control 42, 820-824 (1995).

8. A. D. Kersey and M. J. Marrone, "Bragg grating based nested fibre interferometers," Electron. Lett. 32, 1221-1223 (1996).

9. Z. Wang, F. Shen, L. Song, X. Wang, and A. Wang, "Multiplexed fiber Fabry-Perot interferometer sensors based on ultrashort Bragg gratings," IEEE Photonics Technol. Lett. 19, 622-624 (2007).

10. T. Rossmanith, X. D. Jin, J. S. Sirkis, M. K. Park, V. Venkat, and B. D. Prasad, "Manufacturing of core mirrors for intrinsic Fabry-Perot interferometers using sol-gel process," Proc. SPIE 3670, 34-40 (1999).

11. X. Chen, F. Shen, Z. Wang, Z. Huang, and A. Wang, "Microair-gap based intrinsic Fabry-Perot interferometric fiber-optic sensor," Appl. Opt. 45, 7760-7766 (2006).

12. Z. Huang, Y. Zhu, X. Chen, and A. Wang, "Intrinsic FabryPerot fiber sensor for temperature and strain measurements," IEEE Photonics Technol. Lett. 17, 2403-2405 (2005).

13. V. Bhatia, M. B. Sen, K. A. Murphy, and R. O. Claus, "Wavelength-tracked white light interferometry for highly sensitive 
strain and temperature measurements," Electron. Lett. 32, 247-249 (1996).

14. B. Qi, G. R. Pickrell, J. Xu, P. Zhang, Y. Duan, W. Peng, Z. Huang, W. Huo, H. Xiao, R. G. May, and A. Wang, "Novel data processing techniques for dispersive white light interferometer," Opt. Eng. 42, 3165-3171 (2003).

15. F. Shen and A. Wang, "Frequency-estimation-based signal-processing algorithm for white-light optical fiber Fabry-Perot interferometers," Appl. Opt. 44, 5206-5214 (2005).

16. M. Han, Y. Zhang, F. Shen, G. R. Pickrell, and A. Wang, "Signal-processing algorithm for white-light optical fiber extrinsic Fabry-Perot interferometric sensors," Opt. Lett. 29, 17361738 (2004).

17. M. Han and A. Wang, "Mode power distribution effect in white-light multimode fiber extrinsic Fabry-Perot interferometric sensor systems," Opt. Lett. 31, 1202-1204 (2006).
18. M. Han and A. Wang, "Exact analysis of low-finesse multimode fiber extrinsic Fabry-Perot interferometers," Appl. Opt. 43, 4659-4666 (2004).

19. A. Kumar, R. K. Varshney, C. S. Antony, and P. Sharma, "Transmission characteristics of SMS fiber optic sensor structures," Opt. Commun. 219, 215-219 (2003).

20. S. M. Tripathi, A. Kumar, R. K. Varshney, Y. B. P. Kumar, E. Marin, and J.-P. Meunier, "Strain and temperature sensing characteristics of single-mode-multimode-single-mode structures," J. Lightwave Technol. 27, 2348-2356 (2009).

21. S. M. Tripathi, A. Kumar, E. Marin, and J.-P. Meunier, "Critical wavelength in the transmission spectrum of SMS fiber structure employing $\mathrm{GeO}_{2}$ doped multimode fiber," IEEE Photonics Technol. Lett. 22, 799-801 (2010).

22. F. Shen, Z. Wang, W. Peng, K. Cooper, G. R. Pickrell, and A. Wang, "UV-induced intrinsic Fabry-Perot interferometric sensors and their multiplexing for temperature and strain sensing," Proc. SPIE 6174, 61740D (2006). 